\title{
CORREÇÃO DE MORDIDA CRUZADA ANTERIOR FUNCIONAL COM A TERAPIA DE PISTAS DIRETAS PLANAS: RELATO DE CASO
}

CORRECTION OF FUNCTIONAL ANTERIOR CROSSBITE WITH PLANAS DIRECT TRACKS: A CASE REPORT

\author{
LEANDRo Bielli Rossi \\ Mestrando em Odontologia, Área de Ortodontia, Centro \\ Universitário de Araraquara (UNIARA) \\ Karina Eiras Dela Coleta Pizzol \\ Eloisa Marcantonio BoecK \\ NADIA LunARDI \\ Artenio José IsPer Garbin \\ Doutora em Ortodontia, Professora dos Cursos de Graduação \\ e Pós-graduação em Odontologia_ Departamento de Orto- \\ dontia do Centro Universitário de Araraquara (UNIARA) \\ Doutora em Ortodontia, Professora dos Cursos de Graduação \\ e Pós-graduação em Odontologia_ Departamento de Orto- \\ dontia do Centro Universitário de Araraquara (UNIARA) \\ Doutora em Materiais Dentários, Professora dos Cursos de \\ Graduação e Pós-graduação em Odontologia_ Departamen- \\ to de Ortodontia do Centro Universitário de Araraquara \\ (UNIARA) \\ Livre docente em Gestão em Marketing, Professor adjunto do \\ Departamento Infantil e Social da Faculdade de Odontologia \\ de Araçatuba-UNESP
}

\section{Resumo}

A mordida cruzada anterior caracteriza-se por um relacionamento vestibulolingual anormal entre os incisivos superiores e inferiores, no qual, os dentes anteriores mandibulares encontram-se por vestibular aos dentes anteriores superiores. Quando a mordida cruzada anterior ocorre nas fases das dentaduras decídua ou mista, é coerente a indicação de tratamento precoce, uma vez que a mesma pode causar desgastes na face vestibular dos dentes superiores envolvidos, recessões gengivais nos incisivos inferiores, além de alterar o crescimento normal dos maxilares no sentido ântero-posterior. O diagnóstico correto e o tratamento precoce são fundamentais para se obter equilíbrio oclusal, estético e funcional. As pistas diretas Planas estão indicadas para o tratamento de mordida cruzada anterior funcional pelos conceitos de reabilitação neuro-oclusal. O presente trabalho relatou um caso clínico de uma criança com mordida cruzada anterior funcional na dentadura decídua tratada pela técnica de pistas diretas Planas. Os resultados mostraram a eficácia do método empregado para o tratamento da referida maloclusão.

Palavras-chave: MÁ oclusão, Dentição Primária, Ortopedia.

\section{Abstract}

The anterior crossbite is characterized by an abnormal buccolingual relationship between the upper and lower incisors, in which the mandibular anterior teeth are in the buccal to the maxillary anterior teeth. When the anterior crossbite occurs during the deciduous or mixed dentition, is consistent indicating early treatment, since it can cause wear on the buccal surface of the upper teeth involved, gingival recession in the lower incisors, and to alter the normal growth jaw in the anteroposterior direction. The correct diagnosis and early treatment are essential to obtain occlusal equilibrium, aesthetic and functional. The Planas direct tracks are indicated for the treatment of anterior crossbite in the concepts of functional neuro-occlusal rehabilitation. This paper reported a case of a child with functional anterior crossbite in deciduous dentition treated by the technique of Planas direct tracks. The results show the effectiveness of the method employed to treat said malocclusions.

Keywords: Malocclusion, Dentition, Primary, Orthopedics 


\section{INTRODUÇÃO}

A mordida cruzada anterior refere-se a uma relação vestibulolingual anormal entre incisivos superiores e inferiores, promovendo comprometimento da estética dento-facial e das funções do sistema estomatognático. De acordo com as características clínicas e radiográficas, a mordida cruzada anterior pode ser classificada, segundo Moyers ${ }^{1}$ em 3 tipos: Dentária, Funcional e Esquelética, sendo que o prognóstico e a forma de tratamento divergem entre as mesmas. Sua etiologia é multifatorial, podendo ocorrer por fatores hereditários associados ou não à fatores ambientais, tais como: trauma ou retenção prolongada do dente decíduo, perda dental prematura, presença de dentes supranumerários e/ ou odontomas, hábitos, e parafunções ${ }^{2-5}$.

Assim como a etiologia é diversificada, a frequência e o tipo de mordida cruzada, também apresentam variações nas diferentes faixas etárias. A mordida cruzada anterior funcional, é frequentemente encontrada nas fases das dentaduras decídua e mista, apresentando forte caráter ambiental. Este tipo específico de mordida cruzada, é caracterizada pela protrusão mandibular funcional, causada por uma interferência na trajetória do fechamento mandibular. Há um contato prematuro durante a oclusão cêntrica, levando a criança a adotar uma postura mandibular desviada por acomodação. Quando tal postura protruída da mandíbula não é corrigida precocemente, poderá causar em longo prazo desvios no crescimento e no desenvolvimento da face, os quais podem resultar em assimetria, com agravantes só corrigidos pela cirurgia ortognática.

Embora com agravantes na fase adulta, o prognóstico da intervenção precoce da mordida cruzada anterior funcional é bastante favorável, uma vez que não há comprometimento esquelético nesta fase; da mesma forma, que o tempo de tratamento mostra-se relativamente curto nestes casos. No entanto, é indispensável a realização de um diagnóstico correto, capaz de diferenciar a mordida cruzada causada por uma alteração postural da mandíbula, daquela provocada por um crescimento desproporcional das bases ósseas no plano sagital. O método preconizado para o diagnóstico diferencial é a comparação da oclusão habitual do paciente (MIH) com a oclusão resultante da manipulação mandibular levando os côndilos em relação cêntrica (RC).

O tratamento precoce desta alteração oclusal de acordo com Planas ${ }^{6}$, basea-se na reabilitação neuro-oclusal de pacientes pediátricos. A terapia sugerida pelo autor visa a correção de mordidas cruzadas funcionais, gerando uma mudança na postura mandibular e corrigindo a dinâmica músculo-esquelética até então em má função7.

Para realização da técnica, o profissional deve manipular a mandíbula do paciente, levando seus côndilo em relação cêntrica (RC) a fim de detectar possíveis contactos oclusais prematuros. Nas áreas com interferências oclusais detectadas deve-se realizar desgastes oclusais seletivos, e em seguida, serem confeccionadas pistas em resinas compostas nas faces incisais e/ou oclusais dos dentes da região em cruzamento. O objetivo destas pistas diretas é formar um barreira que impessa o retorno da mandíbula à posição de desvio mandibular a qual estava habituada. ${ }^{8}$

Esta terapia, desenvolvida por Planas desde a década de 70 é utilizada por inúmeros autores ${ }^{8-15}$ como forma de tratamento da mordida cruzada funcional por apresentar algumas vantagens como: baixo custo comparado aos aparelhos ortopédicos e/ou ortodônticos, utiliza a função como estímulo de crescimento, não apresenta riscos de sobrecorreção, é rápida, não depende da colaboração do paciente, e pode ser utilizada em crianças em idade precoce, nas fases das dentaduras decídua ou mista, evitando a instalação de problemas ósseos futuros ${ }^{8,9}$

O artigo relata um caso clínico de uma criança com mordida cruzada anterior funcional tratada pela técnica de pistas diretas Planas estabelecendose a correção da maloclusão.

\section{Caso Clínico}

O paciente PVG, 4 anos e 2 meses, gênero masculino, compareceu à clinica de Ortodontia e Ortopedia Funcional da Associação Brasileira de Odontologia (ABO) regional de Araçatuba, para avaliação ortodôntica. Constatou-se que o mesmo apresentava mordida cruzada anterior, padrão mesofacial, dentadura decídua, ausência de cárie ou lesão periodontal e boas condições de higiene, sendo solicitada a documentação ortodôntica completa. Nenhuma anomalia ou outra alteração oclusal foi observada durante a análise da documentação ortodôntica.

Após exame clínico detalhado, na qual a mandíbula do paciente foi manipulada levando os côndilos em relação cêntrica (RC), classificou-se a mordida cruzada como funcional. Observouse a presença de contatos prematuros entre os incisivos superiores e inferiores, quando em RC, sendo tais interferências responsáveis pela postura anteriorizada da mandíbula estando a oclusão em MIH (Figuras 1A- F). 

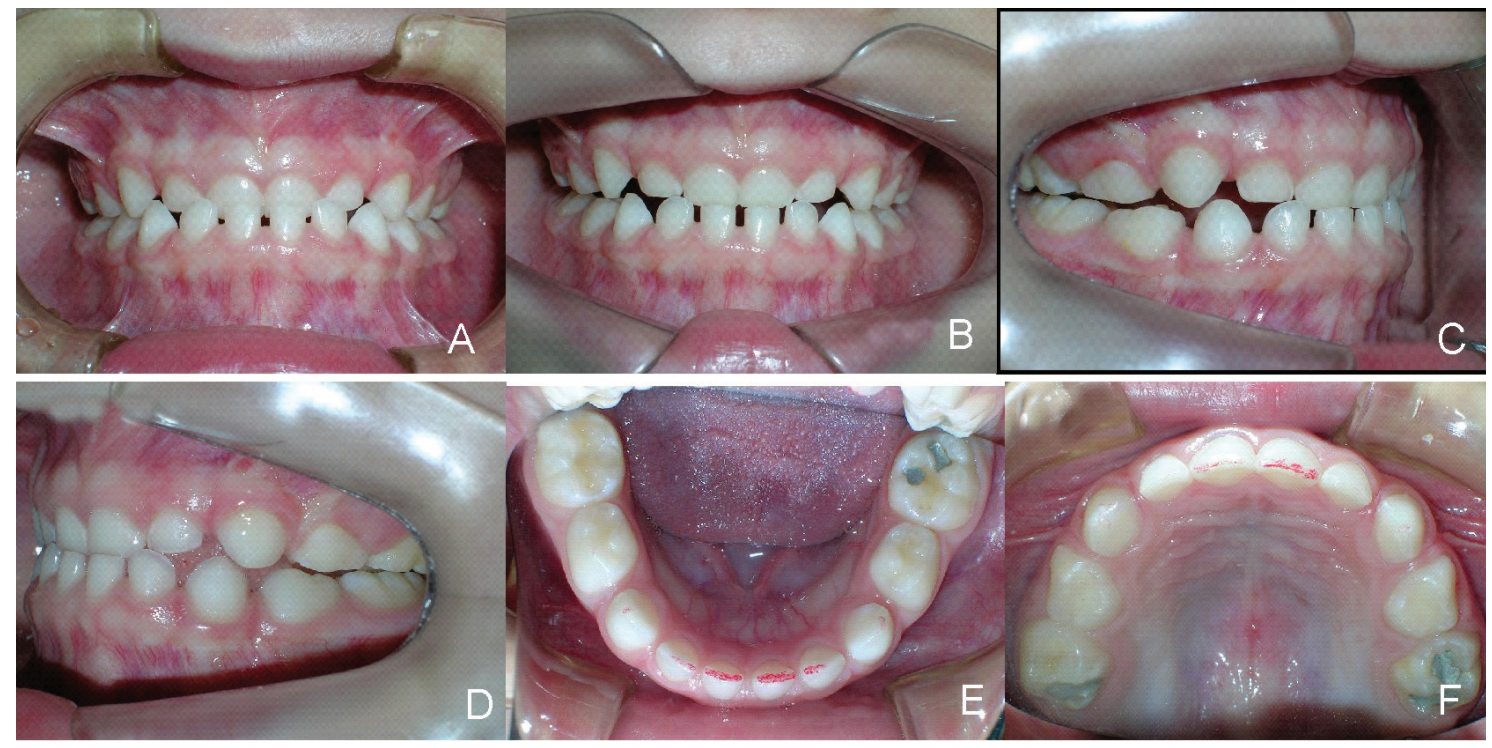

Figuras 1A) Paciente com oclusão em MIH, mostrando a relação de cruzamento dentário anterior; 1B-D) e em relação cêntrica, evidenciando a relação de topo dos dentes anteriores enquanto os posteriores encontram-se desocluídos. As figuras $1 \mathrm{E}$ e $1 \mathrm{~F}$ mostram a presença de contatos prematuros dos incisivos.

Baseado no exame clínico o paciente foi considerado apto à terapia ortopédica de Planas com utilização de pistas diretas confeccionadas sob incisivos superiores.

Os contatos oclusais prematuros foram removidos, de forma a criar uma plano inclinado de aproximadamente $45^{\circ}$ entre a face palatina dos incisivos superiores e a face vestibular dos incisivos inferiores. As pistas diretas Planas foram então confeccionadas em resina composta Z100 (3M) na cor P, após prévio condicionamento com ácido fosfórico $37 \%$ por 30 segundos, lavagem e secagem, e aplicação do adesivo Single Bond (3M) com auxílio de aplicador (Microbrush). O acabamento foi realizado com pontas diamantadas em alta rotação, próprias para acabamento de resina composta, e pontas Enhance (Dentsply); e a distribuição e homogenidade dos pontos de contato foram testados com carbono apropriado

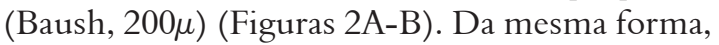
os movimentos de lateralidade foram testados para que os mesmos fossem realizados livremente em ambos os lados e de maneira equilibrada.

O paciente foi orientado quanto aos cuidados necessários para manutenção das pistas, bem como da forma correta de ocluir. Após 14 dias, o paciente retornou à clínica com as pistas fraturadas. Novas pistas foram confeccionadas e por um período de 60 dias elas foram deixadas splintadas e posteriormente, separadas como preconiza a técnica. Esta modificação da técnica teve o intuito de manter e estabilizar as pistas para a adaptação do paciente em sua nova postura mandibular (Figura 3).

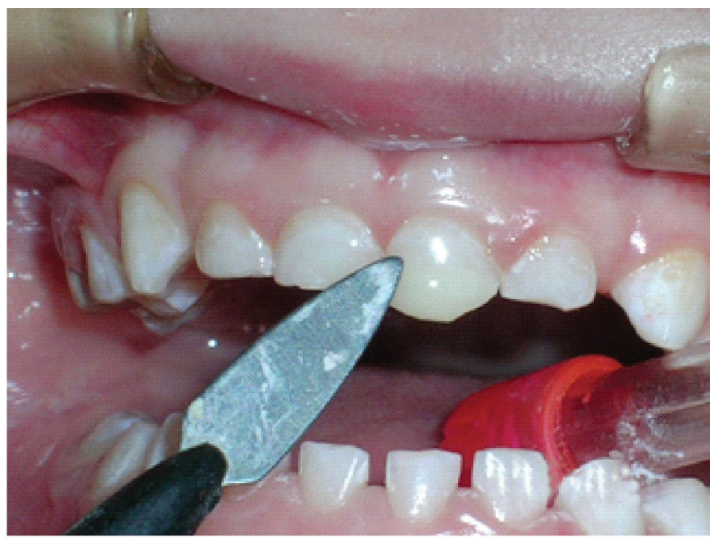

Figura 2 A) Confecção das pistas diretas Planas em resina composta

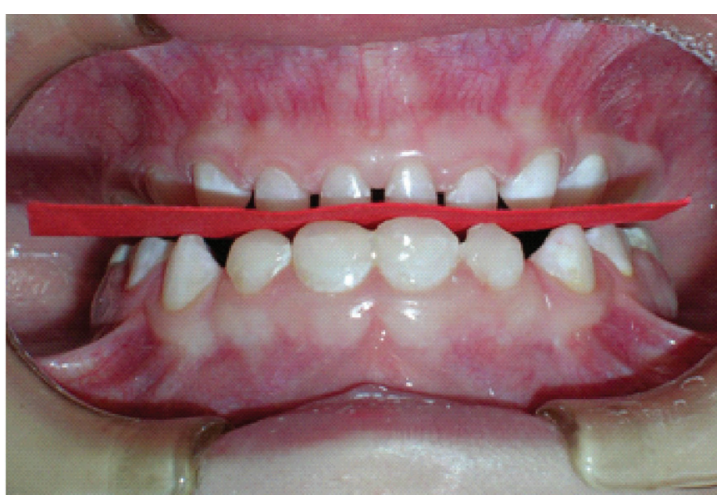

FIGURA 2 B) conferência da distribuição e homogenidade dos pontos de contato com carbono apropriado. 


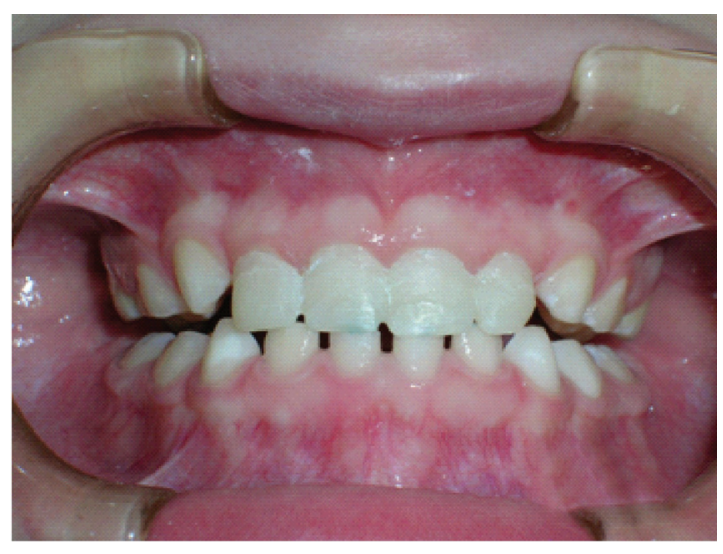

FigURA 3) Foto da oclusão do paciente após a construção de novas pistas splintadas.
A correção completa da mordida cruzada, bem como a obtenção da intercuspidação posterior foram alcançadas após 30 dias do início do tratamento (Figuras 4A-C). Para manter a estabilidade da oclusão obtida, o paciente foi acompanhado durante três meses com retornos mensais.

As pistas diretas foram mantidas mesmo após o descruzamento dos incisivos e retornos bimestrais foram agendados para ajustes e desgastes aleatórios das mesmas, evitando-se limitar e/ou dificultar os movimentos mandibulares, além de acompanhar as trocas dentárias até o estabelecimento da oclusão permanente. Nestes retornos foram avaliados a postura mandibular, os corretos contatos oclusais, o perfil e hábitos do paciente que influenciem negativamente no desenvolvimento das bases ósseas, bem como a necessidade de tratamento ortodôntico ou ortopédico corretivo no futuro (Figuras 5A-D).

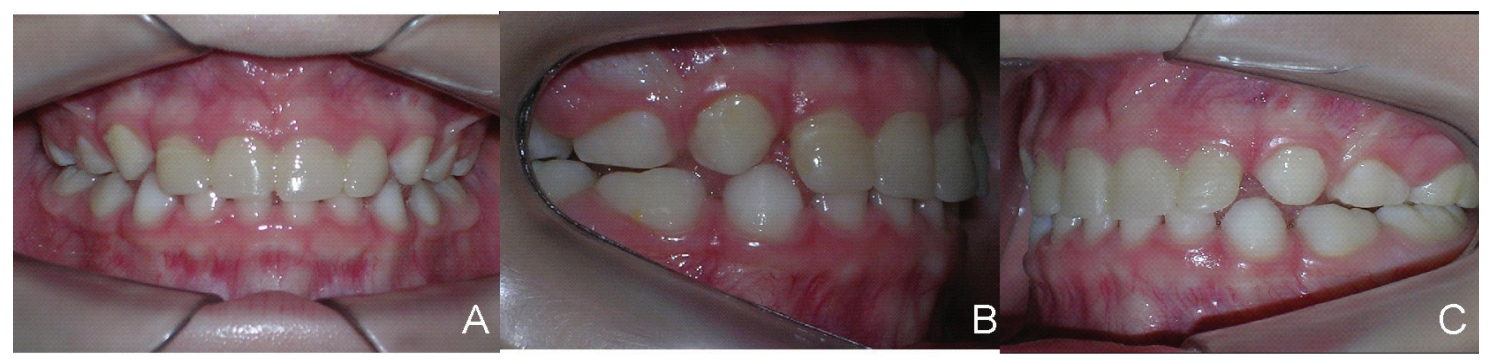

Figuras 4 A-C) Oclusão do paciente após a correção completa da mordida cruzada obtida após 30 dias do início do tratamento.
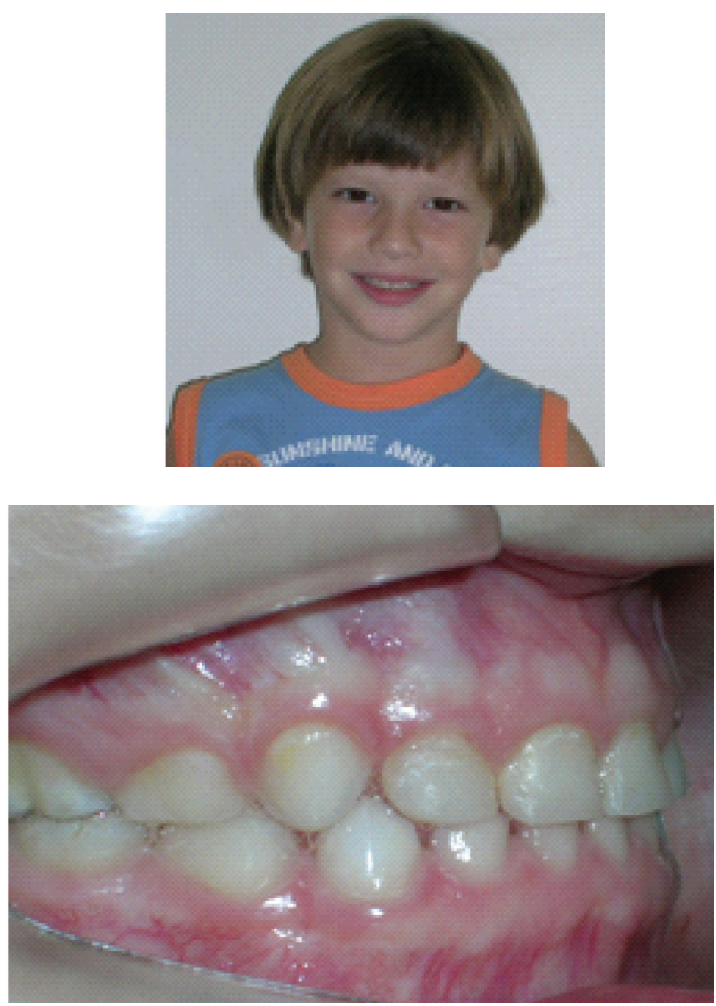
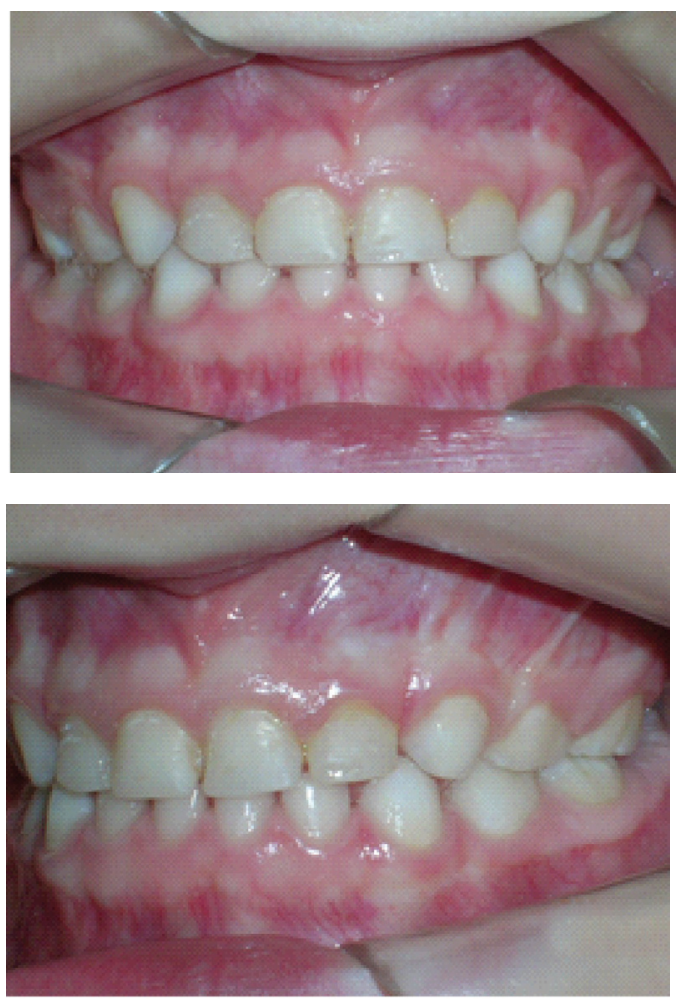

FIGURAS 5A-D) Fotos extra-bucal e intra-bucais do paciente após 8 meses da construção das pistas diretas Planas, durante o período de acompanhamento. 


\section{Discussão}

Segundo o médico e fisiologista Claude Bernard "a função cria o órgão, e o órgão proporciona a função". Complementando-o, Planas ${ }^{7}$ conclui que, se a função parte de uma excitação neural, e esta sendo fisiológica, produzirá um efeito ou desenvolvimento fisiológico. Ao contrário, se a mesma for patológica a resposta do desenvolvimento será da mesma forma, patológica. Fundamentando-se nestes conceitos, Planas ${ }^{7}$ sugeriu que o tratamento de reabilitação neuro oclusal (RNO) deve ter como base descobrir onde, quando e como atuar nos centros neurais receptores que proporcionam a resposta do Sistema Estomatognático, excitando-os fisiológicamente e na medida necessária para obtenção de uma resposta de desenvolvimento normal e equilibrada.

Desta forma, quanto mais precoce for a desprogramação neural e muscular dos efeitos patológicos e conseqüente reabilitação dos sinais fisiológicos, menor será o tempo necessário para a correção da alteração da função do órgão (músculo/dente).

A busca por uma clusão normal e fisiológica, exige do profissional atenção especial quanto as mudanças que ocorrem na oclusão durante a transição da dentadura decídua para a mista, e dessa para a permanente ${ }^{16,17}$. Embora as características de normalidade sejam divergentes entre as arcadas decídua e a permanente no que se refere a presença de diastemas, angulação, inclinação dentária, entre outros aspectos; de forma geral, a oclusão pode ser considerada normal quando os dentes estão corretamente ordenados no arco e em harmonia com todas as forças estáticas e dinâmicas que sobre eles atuam. Segundo Vellini ${ }^{18}$ a oclusão normal é uma oclusão estável, sã e esteticamente atrativa.

Portanto, torna-se fundamental não só ao odontopediatra e ao ortodontista, bem como ao clínico, verificar a presença de condições que possam contribuir para o desenvolvimento de uma oclusão normal e no diagnóstico de desvios de normalidade na dentição em desenvolvimento, pois o diagnóstico e tratamento precoce dessas situações desfavoráveis visam o estabelecimento de uma oclusão permanente fisiológica. ${ }^{19}$

Segundo Santos $^{20}$ as pistas diretas podem ser compreendidas como o acréscimo de resina fotoativa sobre a superfície dos dentes decíduos, em sua face oclusal, de forma individual, respeitando e preservando a unidade biológica do dente.

Seguindo o princípio da reabilitação neurooclusal, as inclinações das pistas devem ser feitas de acordo com as necessidades de cada caso, sempre relacionada com o Plano de Camper e orientadas de acordo com a conveniência da liberação do movimento mandibular e estímulo de crescimento dentro das leis Planas de crescimento maxilomandibular ${ }^{4,713}$. De acordo com este princípio, as pistas diretas Planas estariam indicadas para diferentes situações como: liberar os movimentos mandibulares (como nas sobremordidas); estimular o crescimento pósteroanterior e transversal mandibular; estimular o crescimento póstero-anterior da maxila; estimular o crescimento dento-alveolar transversal da mandíbula e da maxila; sendo as mesmas construídas diferentemente para cada caso ${ }^{21}$.

Entre as principais vantagens da técnica de pistas diretas Planas, pode-se destacar o fato das mesmas não necessitarem da colaboração do paciente, já que são baseadas em "restaurações adesivas" e desgastes seletivos que permanecem atuantes no sistema estomatognático 24 horas por dia; garantem a manutenção da relação intermaxilar correta durante o desempenho de funções ativadoras do crescimento facial como a mastigação, o que é fundamental para o tratamento. A técnica é simples e apresenta baixo custo, uma vez que dispensa material ou equipamento especial para sua realização ${ }^{8}$.

Diversos autores ${ }^{2,10,21-25}$ usam e preconizam a técnica de pistas diretas Planas para casos onde não haja a possibilidade de equilibrar a oclusão apenas através do desgaste seletivo, como também para complementação de tratamento ortopédico funcional convencional (uso de aparatologias removíveis) e para o tratamento de todas as patologias de origem oclusal funcional prevenindo seu agravamento (mordida cruzada unilateral ou bilateral, sobremordida, distoclusão, mordida aberta anterior e mesioclusão), nas dentaduras decídua e/ou mista o mais precocemente possível, obtendo sucesso total ou parcial para correção definitiva da oclusão do paciente.

\section{Conclusão}

O uso da técnica de pistas diretas proposta por Planas permitiu a correção da mordida cruzada anterior funcional após um mês do início do tratamento. Isso mostra que o tratamento das mordidas cruzadas pode e deve ser realizado o mais precocemente possível por profissionais com suficiente conhecimento da fisiologia oclusal e técnica de confecção das pistas em resina. As pistas diretas podem ser parte integrante de uma 
das etapas do tratamento ortodôntico/ortopédico, em pacientes com dentaduras decídua ou mista, propiciando maiores chances de sucesso na reabilitação do sistema estomatognático.

\section{REFERÊNCIAS}

1. Moyers RE. Ortodontia. Do original: Handbook of Orthodontics, 1988. Revisão Técnica por Aloysio Cariello. 4.ed., Rio de Janeiro: Guanabara Koogan; 1991, 483p.

2. Lee BD. Correction of crossbite. Dent Clin North Am 1978 Oct; 22:647-68.

3. Nakasima A, Ichinose M, Nakata S. Genetic and environmental factors in the development of socalled pseudo and true mesiocclusions. Am J Orthod Dentofacial Orthop 1986; 90:106-16.

4. Sári S, Gokalp H., Aras S. Correction of anterior dental crossbite with composite as an inclined plane. Int J Pediatric Dent 2001 May; 11:201-8.

5. Thilander B. Basic mechanisms in craniofacial growth. Acta Odontol Scand 1995 Jun; 53:144-55.

6. Planas P. Importancia del diagnostico, tratamiento precoz en ortodoncia y equilíbrio oclusal. Rev Esp Estomatol 1988 Nov/Deic.; 6(6):477-82.

7. Planas P. Reabilitação neuro-oclusal (RNO). 2.ed. Rio de Janeiro: Ed. Médica e Científica, 1997; 355p.

8. Chibinski ACR., Czlusniak GD, Melo MD. Pistas diretas Planas: terapia ortopédica para correção de mordida cruzada funcional. Clin Ortodon Dental Press 2005; 4(3):64-72.

9. Almeida GP. Ortopedia Funcional dos maxilares na dentição decídua. Rev Odontologia Ensino e Pesquisa 1997; 2(2):17-23.

10. Arns OH, Ortellado G. Pistas diretas planas para a correção de mordida cruzada posterior. JBO 2001; 31:15-9.

11. Bertele GP. Correção de desvio Lateral funcional na dentição decídua: uso do método original de Pedro Planas. Mondo Ortod 1982 Sept./Oct; 7:7-14.

12. Brandão MRC. Pista Direta Planas na Correção da Mordida Cruzada Posterior. Revista da APCD 1995 Mar./Abr.; 49(2):127-9.
13. Gribel M N. Planas direct tracks in the early treatment of unilateral crossbite with mandibular postural deviation. Why worry so soon? World J Orthod 2002; 3:239-49.

14. Simões W A. Ortopedia funcional dos maxilares vista através da reabilitação neuro-oclusal. São Paulo: Ed. Santos, 1985, p.167-173.

15. Tashima AY, Verrastro AP, Ferreira SLM., Wanderley MT, Guedes-Pinto E. Tratamento Ortodôntico Precoce da Mordida Cruzada Anterior e Posterior: Relato de caso clínico. JBP 2002; 6(29):24-31.

16. Baume LJ. Physiological tooth migration and its significance for the development of occlusion. I. The biogenetic course of the deciduous dentition. J Dent Res 1950; 29(2):123-32.

17. Moura MS, Simplício AHM, Moura LFAD, Moura WL. Alterações na relação molar entre as dentaduras decídua e mista. Rev ABO 1994; 2(5):333-39.

18. Vellini F. Ortodontia: diagnostic e planejamento clinico. São Paulo: Artes Médicas, $2^{\mathrm{a}}$. ed. 1998.503 p.

19. Nanda RS, Khan I, Anand R. Age changes in the occlusal pattern of deciduous dentition. J Dent Res 1973; 52(2):221-4.

20. Santos JLB. Como resolver pequenos problemas ortodônticos sem o auxílio do especialista. In: Todescan FF, Bottino MA, coordenadores. Atualização na clínica odontológica: a prática da clínica geral. São Paulo: Artes Médicas, p. 519-39.1996

21. Neto GP, Puppin-Rontani RM, Garcia RCMR. Changes in the masticatory cycle after treatment of posterior crossbite in children aged 4 to 5 years. Am J Orthod Dentofacial Orthop 2007; 131(4):464-72.

22. Canalda K. Homage to Pedro Planas. Actual Odontostomatol 1992; 172:561-8.

23. Gribel MN, Gribel BF. Planas direct tracks in young patients with Class II malocclusion. World J Orthod 2005; 6(4):355-68.

24. Limme M. Interception in the primary dentition: mastication and neuro-occlusal rehabilitation. Orthod Fr 2006; 77(1):113-35.

25. Simões WA, Petrovic A, Stutzmann J. Modus operandi of Planas' appliance. J Clin Pediatr Dent 1992:16(2):79-85.

Submetido em: 19-9-2012

Aceito em: 28-1-2013 